\title{
Interferometry for High Resolution Absolute Distance Measuring by Larger Distances
}

\author{
E. Dalhoff, E. Fischer, S.Kreuz, H.J. Tiziani \\ Universität Stuttgart, Institut für Technische Optik \\ Pfaffenwaldring 9, D- 7000 Stuttgart 80
}

\section{Introduction}

There are some techniques of absolute remote distance measurement. Some of it deserve the title of absolutness without restriction (triangulation e.g.) but are not useful measuring at distances of some ten metres because of practical reasons. The other techniques have an unambiguity range within the measurement is absolute. Among the incoherent techniques the phase measurement of modulated light seem to have reached some limitation with an resolution of less than $1 \mathrm{~mm}$. This limitation is set by the bandwidth of the photodetector. The coherent techniques operate with two wavelengths to extend the unambiguity range of the classical interferometry. The unambiguity range is then half the synthetic wavelength and can be adjusted quite arbitrarily from some ten micrometers on. If heterodyne detection is involved a relative resolution of the electronic phase measurement of $10^{-4}$ is achievable. As is valid for the phase measurement of intensity modulated light cascading of 2 or more stages of different synthetic wavelength is necessary in order to improve the relative resolution of the whole system.

The main advantage of the coherent technique is that a resolution below 1 micron is possible. In addition the signal-to-noise ratio (SNR) makes the effort of coherent detection attractive especially working at large distance. In Fig. 1 the SNR of the detector signal is depicted for the coherent system discussed in this paper and the incoherent phase measuring technique depending on the power of the light backreflected by the object.

In the following a set-up of a double heterodyne interferometer with an unambiguity range of 100 metres and a resolution of $0.1 \mathrm{~mm}$ (corresponding to a relative resolution of $10^{-6}$ ) will be described and experimental results will be presented.

\section{Set-Up}

The set-up is designed to yield a resolution of $0.1 \mathrm{~mm}$ with an unambiguity range of 100 metres. A sketch of it is shown in Fig.2. Light of a monomode laser diode with wavelength $\lambda_{1}$ is frequencyshifted by a $500 \mathrm{MHz}$ acousto-optic modulator (AOM) in order to yield the second wavelength $\lambda_{2}$. This corresponds to a synthetic wavelength of $60 \mathrm{~cm}$. Light of the wavelengths $\lambda_{1}, \lambda_{2}$ is used as reference 


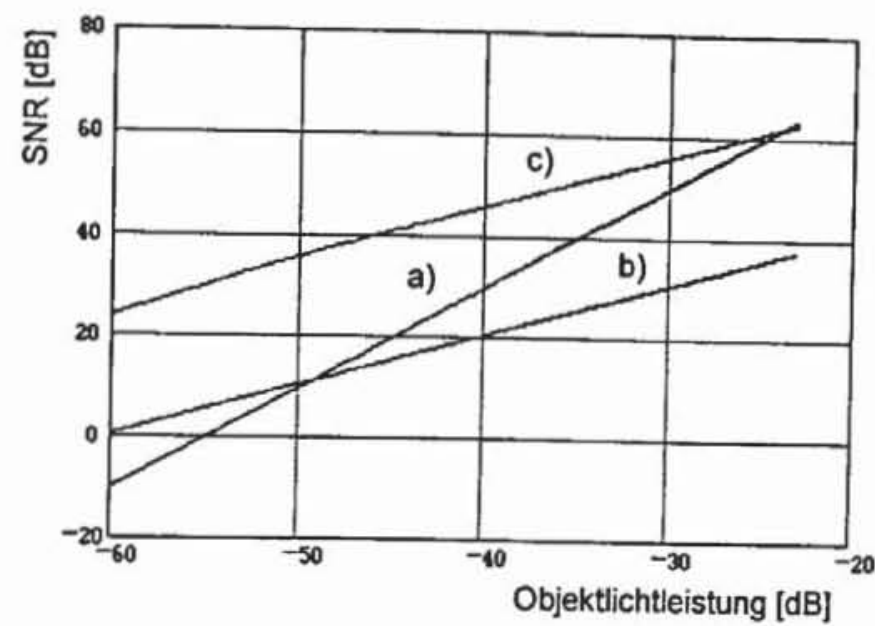

Fig.1 SNR dependent on object light power for a) incoherent phase measuring, b) coherent heterodyne detection on one wavelength (BW $15 \mathrm{MHz}$ ), c) double heterodyne detection (BW $15 \mathrm{MHz}$ ).

light, while the object light is shifted by means of two additional AOM with $f 1=80$ resp. $f 2=80.1$ $\mathrm{MHz}$ in order to provide double heterodyne reception /1/. The object and reference light gets superimposed on a photodetector. Dropping the dc-component the signal after demodulation is given by

$$
i_{\text {det }}=\left|i_{\text {det }}\right| \cdot \cos \left(2 \pi \cdot\left(\Delta f \cdot t+\frac{2 z}{\Lambda}\right)\right)
$$

where $\Delta f=\left|f_{1}-f_{2}\right|$ and $\Lambda=\left(\lambda_{1} \cdot \lambda_{2}\right) /\left|\lambda_{1}-\lambda_{2}\right| . Z$ is the distance to be measured. Thus $\Delta f=100 \mathrm{kHz}$ is the heterodyne frequency of the demodulated signal and the unambiguity range of the measurement is given half the synthetic wavelength $\Lambda / 2=30 \mathrm{~cm}$. The distance is evaluated from a measurement of the phase of the heterodyne signals of a measuring and a control interferometer with fixed paths. The extended unambiguity range of 100 metres is obtained by computing the results of two measurements with slightly different synthetical wavelength $\Lambda=58.9 \mathrm{~cm}$ corresponding to a frequency shift of the AOM of $501.5 \mathrm{MHz}$. In order to maintain the phase resolution of $2 \pi / 3600$ after more than 300 cycles of the unambiguity range, the relative stability of the synthetic wavelength has to be better than $10^{-6}$. Rewriting eq. 1 to $\Lambda=c / \Delta v$ (where $\Delta v$ denotes the frequency difference between the two wavelengths) it can be seen that in this set-up the stability of the phase is depending only on the frequency stability of the AOM and not on the frequency stability of the laser diode. This is an advantage of this approach since a relative stability of an electronic oscillator of better than $10^{-6}$ is state of the art.

In addition to the unavoidable noise in the detection process the decorrelation of the two speckle patterns created by the object light when measuring at rough surfaces causes a phase error $/ 2 /$. The standard deviation of this statistical phase error depends on the wavelength difference, the roughness of the target and its tilt. Measuring at a perpendicular oriented surface with a mean roughness of $10 \mu \mathrm{m}$ it is calculated to be below $0.1 \mathrm{~mm}$ for the set-up in question. This phase error increases when a misalign- 
ment between the object light of the two wavelengths occurs. Therefore the object and reference light after the 80 resp. $80.1 \mathrm{MHz} \mathrm{AOM}$ is fed into two monomode fibres where couplers split the light for the control interferometer. The fibres guarantee the alignment of the light of the two wavelengths.

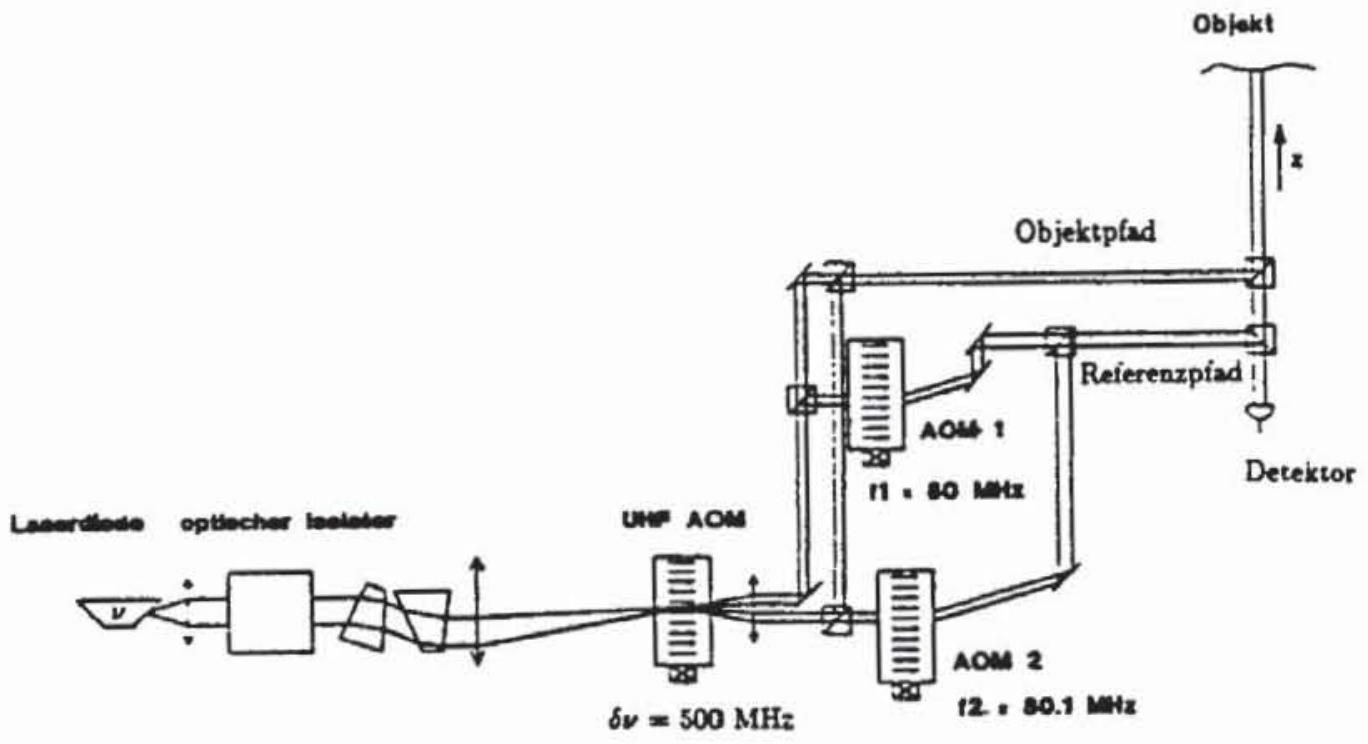

Fig.2: Double heterodyne interferometer set-up.

\section{Results}

Fig. 3 shows a $30 \mathrm{~s}$ stability measurement at a rough surface at $20 \mathrm{~m}$ distance. The resolution is 0.08 $\mathrm{mm}$. By now systematic errors reduce the accuracy to about $1 \mathrm{~mm}$. Fig. 4 shows a measurement at 100 metres distance at a retroreflector with an rms-phase-deviation of $0.2 \mathrm{~mm}$. It shows, that a stable phase measurement is obtained at a distance the optical path length of which is about 40 times the coherence length of the laserdiode used.

Literatur:

I1/ Z.Sodnik, E.Fischer, Th.Ittner u. H.J.Tiziani: Two-wavelength double heterodyne interferometry using a matched grating technique.

Applied Optics, Vol.30 No.22, 1991

121 Vry,U. et.al.: Higher order statistical properties of speckle fileds and their application to rough surface interferometry

Journal of the Optical Society of America, Vol .3 No.7, 1986 
Stabilitâtsmessung

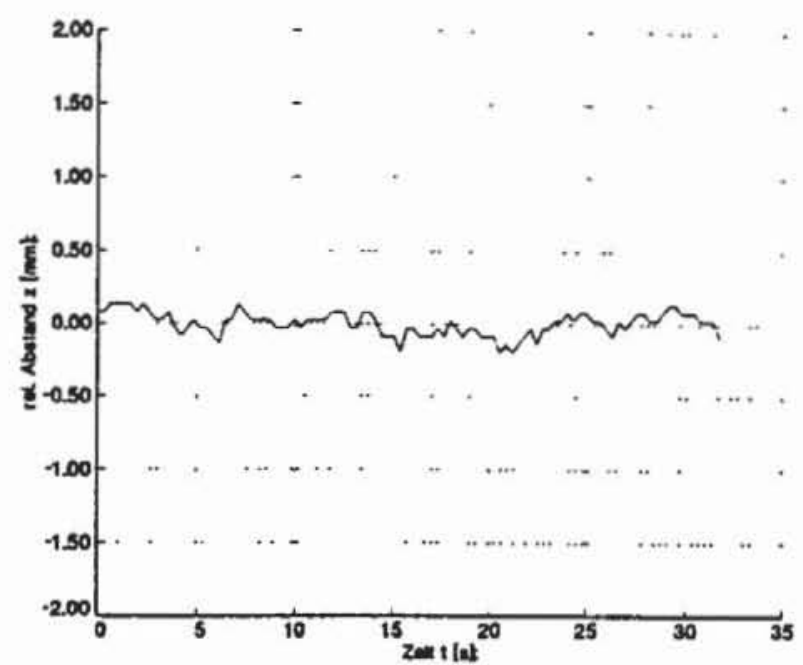

Fig. 3: Stability measurement at a rough target at 5 metres distance. $\Delta z_{r m s}=0.08 \mathrm{~mm}$

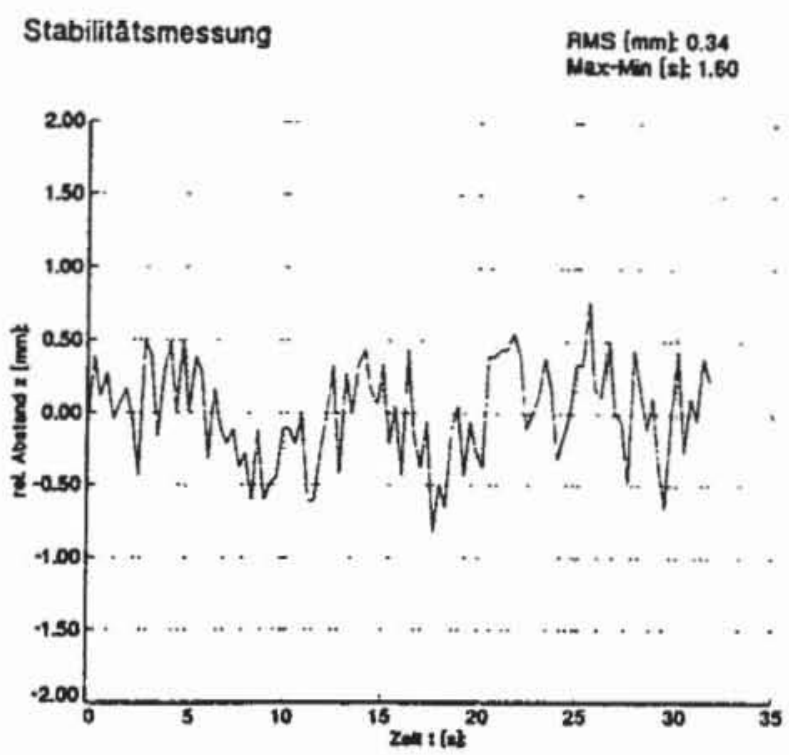

Fig.4: Stability measurement at a cornercube at 100 metres distance. $\Delta \mathrm{z}_{\mathrm{rms}}=0.34 \mathrm{~mm}$. 